\title{
Development of staffing, workload and infrastructure in member departments of the European Organisation for Research and Treatment of Cancer (EORTC) radiation oncology group
}

Willmann, Jonas ; Poortmans, Philip ; Monti, Angelo Fillipo ; Grant, Warren ; Clementel, Enrico ; Corning, Coreen ; Reynaert, Nick ; Hurkmans, Coen W ; Andratschke, Nicolaus

\begin{abstract}
Purpose The EORTC Radiation Oncology Group uses a Facility Questionnaire (FQ) to collect information from its member radiation oncology departments. We analysed the FQ database for patientrelated workload, staffing levels and infrastructure to determine developments in radiation oncology departments in the clinical trials community. Materials Methods We exported the FQ database in August 2019. Departments were included if their FQ was created or updated within the two preceding years. Observations were compared with previous evaluations of the FQ database. Results In total, 161 departments from 24 mostly European countries were analysed. The average number of patients per department increased by $3.0 \%$ to 2,453 (2013: 2,381). The annual number of patients decreased by $7.4 \%$ to 225 per radiation oncologist (2013: 243) and by $7.9 \%$ to 326 per medical physicist (2013: 354$)$. In contrast, the number of patients increased by $23.3 \%$ to 106 per radiation therapist (RTT) (2013: 86) and per treatment unit by $3.9 \%$ to 485 (2013: 467). In a pairwise comparison of departments that were available in 2013 and 2019, the number of patients per radiation oncologist $(\mathrm{p}=0.02)$ and per physicist $(\mathrm{p}=0.0003)$ decreased significantly. The number of departments that own a dedicated PET-CT scanner more than doubled (2013: 4\%; 2019: 9\%) and the availability of stereotactic body radiation therapy (SBRT) increased by $31.8 \%$ to $85.7 \%$ of the departments (2013: 65\%). Conclusion The case-related workload per radiation oncologist and per physicist continues to decrease but increases per RTT and treatment unit. This is likely driven by an increased use of complex techniques, multimodality imaging and the implementation of automation in radiation oncology departments.
\end{abstract}

DOI: https://doi.org/10.1016/j.radonc.2020.11.009

Posted at the Zurich Open Repository and Archive, University of Zurich ZORA URL: https://doi.org/10.5167/uzh-193842

Journal Article

Accepted Version

Originally published at:

Willmann, Jonas; Poortmans, Philip; Monti, Angelo Fillipo; Grant, Warren; Clementel, Enrico; Corning, Coreen; Reynaert, Nick; Hurkmans, Coen W; Andratschke, Nicolaus (2021). Development of staffing, workload and infrastructure in member departments of the European Organisation for Research and Treatment of Cancer (EORTC) radiation oncology group. Radiotherapy and Oncology, 155:226-231.

DOI: https://doi.org/10.1016/j.radonc.2020.11.009 


\section{Title}

Development of Staffing, Workload and Infrastructure in Member Departments of the European Organisation for Research and Treatment of Cancer (EORTC) Radiation Oncology Group

\section{Authors}

Jonas Willmann ${ }^{\text {a1*}}$, Philip Poortmans ${ }^{\text {b,c }}$, Angelo Fillipo Monti ${ }^{\mathrm{d}}$, Warren Grant ${ }^{\mathrm{e}}$, Enrico Clementel a, Coreen Corning a, Nick Reynaert ${ }^{\mathrm{f}}$, Coen W. Hurkmans g and Nicolaus Andratschke ${ }^{\mathrm{h}}$

${ }^{a}$ EORTC Headquarters, Av. E. Mounier 83/11, 1200 Brussels, Belgium

b Iridium Kankernetwerk, Oosterveldlaan 22, 2610 Wilrijk-Antwerp, Belgium

${ }^{c}$ University of Antwerp, Faculty of Medicine and Health Sciences, Campus Drie Eiken, Building S, Universiteitsplein 1, 2610 Wilrijk-Antwerp, Belgium

d ASST GOM Niguarda, Department of Medical Physics, Piazza Ospedale Maggiore 3, 20162 Milan, Italy

${ }^{\mathrm{e}}$ Gloucestershire Oncology Centre, Cheltenham General Hospital, Cheltenham, GL53 7AN, United Kingdom

${ }^{\mathrm{f}}$ Department of Medical Physics, Institut Jules Bordet, Boulevard de Waterloo 121, 1000 Brussels, Belgium

g Department of Radiation Oncology, Catharina Hospital, Michelangelolaan 2, Po box 1350, 5602 ZA Eindhoven, The Netherlands

h Department of Radiation Oncology, University Hospital Zurich, Rämistrasse 100, 8091 Zurich, Switzerland

1 Permanent address: Department of Radiation Oncology, University Hospital Zurich, Rämistrasse 100, 8091 Zurich, Switzerland

* Corresponding author: Department of Radiation Oncology, University Hospital Zurich, Rämistrasse 100, 8091 Zurich, Switzerland. jonas.willmann@usz.ch (J. Willmann).

\section{Acknowledgements}

The development of this project has been supported by Kom op tegen Kanker through the Emmanuel van der Schueren Fellowship for Quality Assurance in Radiation Therapy granted 
in $2019 / 2020$. This research did not receive any specific grant from funding agencies in the public, commercial, or not-for-profit sectors.

\begin{abstract}
Purpose

The EORTC Radiation Oncology Group uses a Facility Questionnaire (FQ) to collect information from its member radiation oncology departments. We analysed the FQ database for patient-related workload, staffing levels and infrastructure to determine developments in radiation oncology departments in the clinical trials community.
\end{abstract}

\title{
Materials \& Methods
}

We exported the FQ database in August 2019. Departments were included if their FQ was created or updated within the two preceding years. Observations were compared with previous evaluations of the FQ database.

Results

In total, 161 departments from 24 mostly European countries were analysed. The average number of patients per department increased by $3.0 \%$ to 2,453 (2013: 2,381). The annual number of patients decreased by $7.4 \%$ to 225 per radiation oncologist (2013: 243) and by 7.9\% to 326 per medical physicist (2013: 354). In contrast, the number of patients increased by $23.3 \%$ to 106 per radiation therapist (RTT) (2013: 86) and per treatment unit by $3.9 \%$ to 485 (2013: 467). In a pairwise comparison of departments that were available in 2013 and 2019, the number of patients per radiation oncologist $(\mathrm{p}=0.02)$ and per physicist $(\mathrm{p}=0.0003)$ decreased significantly. The number of departments that own a dedicated PET-CT scanner more than doubled (2013: 4\%; 2019: 9\%) and the availability of stereotactic body radiation therapy (SBRT) increased by $31.8 \%$ to $85.7 \%$ of the departments (2013: $65 \%$ ).

\section{Conclusion}

The case-related workload per radiation oncologist and per physicist continues to decrease but increases per RTT and treatment unit. This is likely driven by an increased use of complex techniques, multimodality imaging and the implementation of automation in radiation oncology departments.

\section{Keywords}

Radiotherapy, Patient volume, Staffing, Infrastructure, Workload, Quality Assurance 


\section{Introduction}

The European Organisation for Research and Treatment of Cancer (EORTC) Radiation Oncology Group (ROG) connects a network of radiation oncology departments in Europe and beyond to conduct clinical trials across different types of cancer. Besides the initiation of radiation therapy-based clinical trials, the EORTC ROG runs an extensive radiation therapy quality assurance (RTQA) program to ensure consistent and reliable delivery of radiation therapy in clinical trials $(1,2)$. As part of the RTQA program, the EORTC ROG collects the site-related information of its member departments with a dedicated Facility Questionnaire (FQ) to assess if they meet a set of minimum requirements for entering patients in clinical trials. The FQ covers patient volume, staffing levels and information on radiation therapy infrastructure, including data acquisition, treatment planning, patient immobilization and verification, treatment delivery and quality assurance. A PDF version of the current FQ is available online (3). Departments are asked to update their FQ every two years or sooner if new equipment is installed or major changes in staffing occur. The minimum requirements of human resources, equipment and equipment-based workload for entering patients in clinical trials are periodically adjusted to account for general developments in radiation oncology departments and available online (4). These developments are assessed in comprehensive reports of the radiation oncology departments in the FQ database. Previous reports covered the content of the FQ database in the years 1992 (5), 2007 (6) and 2013 (7). From the reports in 2007 to 2013, decreasing numbers of patients per radiation oncologist, per medical physicist and per radiation therapist (RTT) were observed, despite an increasing number of patients per radiation oncology department. During the same time, more departments began introducing new radiation therapy techniques, such as intensity-modulated radiation therapy (IMRT) and stereotactic body radiation therapy (SBRT).

Here, we report the current patient volume, staffing levels and infrastructure in EORTC ROG member radiation oncology departments and show developments compared to previous reports of the FQ database. We also discuss the implications of updating the recommendations on staffing and infrastructure requirements, for predicting demands in radiation oncology departments.

\section{Material \& Methods}

The web-based FQ is filled out and submitted by EORTC ROG member radiation oncology departments through the EORTC QA platform (8). The information is then reviewed by a radiation oncologist and a medical physicist to verify whether the minimum requirements for entering patients in clinical trials are met by the department. If needed, specification of missing 
or unclear data are requested before approval. In June 2019, we asked EORTC ROG member radiation oncology departments whose FQ had expired to submit an updated version. The FQ expires after two years or if there are any significant changes of staffing or the simulation process, treatment planning or delivery process. Data from the FQ database was extracted in mid-August 2019. Departments were analysed if their FQ was valid, i.e. created or updated between August 2017 and August 2019. All FQs in this time window were included, regardless of their approval status. All data are as reported by the responsible person for the member department.

To determine the patient-related workload in radiation oncology departments, the number of patients per year was analysed in relation to staffing levels in the different professional groups and equipment in each department. The ratio of patients per staff in professional groups was used as a surrogate for the actual workload, since specific information on the tasks performed by the different professional groups was not available in our dataset. The staffing levels in the present analysis are reported as full time equivalent (FTE). Patient numbers include both new and retreated patients.

In order to allow for a meaningful assessment of developments in workload, we performed a pairwise analysis of departments that were available in 2013 and 2019 to account for potentially differing compositions of the radiation oncology departments included in the survey. A Wilcoxon matched-pairs signed rank test for paired nonparametric data with a two-sided $\alpha=$ 0.05 was applied to determine statistically significant differences.

Additionally, we determined the proportion of radiation oncology departments in each country that the EORTC departments comprise to determine the representativeness of the presented data. The Directory of Radiotherapy Centres (DIRAC) database by the International Atomic Agency (IAEA) was queried for the current number of radiation therapy departments in each participating country (9). The DIRAC database is continuously updated, based on a questionnaire circulated by the IAEA among its member states. The collected data undergo a process of data review and verification for any inconsistencies and completeness by the IAEA. The data was processed and analysed at the EORTC Headquarters.

\section{Results}

Information on patient volume, staffing levels and infrastructure from 161 radiation oncology departments was analysed for this study, an increase by 3.2\% from the 156 departments in 2013. Ninety-eight departments (61\%) were university hospital (either public or private), while 50 departments $(31 \%)$ were public non-university hospitals. Thirteen departments (8\%) were private, non-university centres. Expectedly, the vast majority of departments (94\%) enrolled 
patients in clinical trials, including but not limited to EORTC trials. The departments were located in 24 countries in Europe, North America, Asia, Africa and Australia. Figure 1 shows the average number of annually treated patients per department in each country, the total number of departments and the numbers of departments that were included in this survey, and the proportion of the departments that were participating from the total number of departments in each country (Fig. 1). A summary table can be found in the Supplementary Materials (Supplementary table 1).

The average number of patients per department per year increased by $3 \%$, from 2,381 to 2,453 (Fig. 2A) as compared to 2013. We observed a decreasing number of patients per radiation oncologist (-7.4\% from 242 to 225) and medical physicist (-7.9\% from 354 to 326) (Fig. 2B). In contrast, the numbers of patients per radiation therapist (RTT) (+23.3\% from 86 to 106) and per treatment unit $(+3.9 \%$ from 467 to 485$)$ both increased. The mean number of treatment units per department remained relatively stable at 5.2 (2012: 5.3). Table 1 summarizes the evolution of patient numbers, staffing levels and workload from 1992 to 2019.

A pairwise analysis of staffing and workload levels was performed on the 75 departments that were available in the 2013 and 2019 survey. In this subset of the departments, we observed a statistically significant decrease of the mean number of patients per radiation oncologist $(-8.5 \%$ from 234 to $214, \mathrm{p}=0.02$ ). The number of patients per medical physicist also decreased significantly $(-12.6 \%$ from 374 to $327, \mathrm{p}=0.0003)$. At the same time, there was a nonstatistically significant decrease of the mean number of patients per RTT ( $-5.6 \%$ from 90 to 85 , $\mathrm{p}=0.33)$ and decrease per treatment unit $(-7.4 \%$ from 489 to $453, \mathrm{p}=0.26)$.

The complexity of modern radiation therapy might account for the changes in staffing and workload of the radiation oncology departments. Precise image-guidance and stereotactic radiotherapy rely on extensive quality assurance and meticulous delineation of target volumes and organs at risk. At the same time, automation might be implemented in steps of the workflow to different degrees, affecting the responsible staff group. To determine potential causes of the observed developments, we assessed the availability of advanced radiation therapy techniques, such as SBRT, stereotactic radiosurgery (SRS), IMRT and volumetric modulated arc therapy (VMAT), as well as imaging modalities, such as MRI or PET-CT. We found that more radiation oncology departments were applying advanced radiation therapy techniques and imaging devices. Since 2013, the number of departments that deliver SBRT increased by $33 \%$ to $86 \%$ of the departments (2013: 65\%) (Fig. 2C). Cranial fractionated SRT and single fraction SRS were widely available in $76 \%$ and $41 \%$ of the departments, respectively (previously not reported). IMRT remains an established standard technique and available in the majority of the departments (2013: 94\%; 2019: 95\%). VMAT, which has first been introduced in 2007 (10), 
was available in $81 \%$ of departments (previously not reported). The number of radiation oncology departments that own a dedicated PET-CT scanner more than doubled (2013: 4\%; 2019: 9\%), while the number of departments owning an MRI scanner remained constant (15\%). The EORTC ROG minimum requirements for staffing and infrastructure in clinical trials including radiation therapy have been consecutively adjusted over the last two decades in order to account for developments that were observed in previous analyses of the FQ database. Table 2 summarizes the changing recommendations from 1992 to 2014 . We determined the number of departments that do not meet the current minimum requirements for staffing and infrastructure. Compared to 2013, fewer sites failed to meet the minimum requirements for workload and infrastructure: $17 \%$ of the departments exceeded the recommended maximum 300 patients per radiation oncologist (2013: 22\%); 7\% exceeded the recommended maximum number of 500 patients per medical physicist (2013: 15\%); $8 \%$ of the departments treated more than the recommended maximum number of 700 patients per treatment unit (2013: 13\%).

\section{Discussion}

The developments of patient volume, staffing and infrastructure in this large, international group of radiation oncology departments complement the trends observed in previous FQ reports. Our report will help updating the recommendations for radiation therapy resources and estimating future requirements.

The number of patients per radiation oncology department is growing in line with predictions from the ESTRO-HERO project. The ESTRO-HERO project aimed to provide a knowledge base for the needs of radiation therapy in Europe to improve resource planning and funding (11). Borras and colleagues predicted a $16.1 \%$ increase of the number of patients that would have an indication for radiation therapy from 2012 to 2025 (12). This corresponds to an annual increase of the patient volume by $1.2 \%$. Applying this prediction to the average number of 2,381 patients per radiation oncology department in 2013, observed in the previous report of the FQ database (7), the predicted number of patients per department at the time of this report would be 2,558 . This prediction is notably close to the observed average of 2,453 patients per department in this report.

The trend towards decreasing numbers of patients per radiation oncologist and per medical physicist - despite growing patient numbers - has also been observed in previous reports of the FQ database (6,7). At the same time, we observed a wider availability of more accurate, potentially less toxic, but time-demanding radiation therapy techniques in radiation oncology departments. These techniques also rely on multimodality imaging, including PET-CET and MRI that are co-registered with traditional planning CT images for precise target volume 
definition. The safe implementation of complex radiation therapy techniques, such as IMRT, VMAT and SBRT, is particularly dependent on highly-skilled radiation oncologists and medical physicist (13) and may thus explain the decreasing number of patients per profession with these developments. Hypofractionated treatments for breast (14), prostate cancer (15), SBRT for early stage lung cancer (16) and (oligo)metastatic disease (17) are becoming the recommended treatment schemes for patient convenience and potential cost advantages with no loss of efficacy and acceptable toxicity. Consequently, reduced treatment fractions per patient might explain the observed higher number of patients per RTT and per treatment unit per year. Additionally, tasks commonly performed by RTTs and dosimetrists might already be more influenced by the implementation of automation in the radiotherapy workflow. For instance, the time for delineation of organs at risk may be significantly reduced by the application of autosegmentation tools $(18,19)$, thus allowing for a higher number of patients per RTT in centres where RTTs perform contouring. Importantly, the increased number of patients per RTT and treatment unit must be balanced by higher educational requirements, skills, and quality assurance as the RTTs are ultimately the professionals who deliver the treatment to the patient.

Fewer sites exceeded the minimum requirements for workload and infrastructure to enrol patients in radiation therapy-based clinical trials of the EORTC. Similar developments were shown in an ESTRO-HERO report (20): the average staffing levels in European radiation oncology departments in 2014 were consistent with, or even more favourable than the QUARTS recommendations from 2005 (21,22). The reasons for this observation may be manifold. Staffing and workload guidelines by international organisations may serve as a benchmark for optimizing the organisation in departments and allocating new investments. The periodic adjustment of guidelines, such as the EORTC minimum requirements, might therefore directly impact the trends observed in this survey.

On the other hand, adequately accounting for the impact of technological developments in radiation oncology departments - from evolving fractionation schemes to changing time demands and professional roles - might be challenging to incorporate into workload and staffing recommendations. Potential professional deficits arising from growing patient volumes and more time-consuming treatments might thus remain undetected. Dunscombe and colleagues found that the actual number of courses per radiation oncologist and medical physicist were even lower than the ESTRO-HERO predictions and the QUARTS guidelines in three quarters of the investigated European countries, suggesting that clinical developments are outpacing guideline developments (23). Similarly, we found a decreasing proportion of departments exceeding the maximum number of patients per radiation oncologist and per 
medical physicist at 17\% and 7\%, respectively. This might indicate that European guidelines including those from the EORTC - may not sufficiently take into account the rapid technological evolution in radiation therapy. Inadequate staffing levels will hamper the ability to deliver safe and high-quality radiation therapy, particularly as technology is constantly evolving and implemented into clinical practice. Commonly used guidelines for staffing and infrastructure of radiation oncology departments include QUARTS (22) and recommendations from other organisations such as IAEA (24), EFOMP (25), IPEM (26) and the EORTC itself (4). The IAEA provides the most comprehensive activity-based approach to predict staffing requirements in radiation oncology departments. The quantitative algorithm captures relevant activities across the entire radiation therapy workflow, related to patients, equipment, education and non-clinical activities to predict the required staffing levels of the different radiation oncology professions. The output reflects the level of technological complexity and the services implemented. Of note, advanced technologies including SBRT, particle therapy, robotic radiation therapy and MR-linac treatments are not included in this algorithm.

Staffing recommendation strategies also differ among radiation oncology professions and might therefore affect staffing developments. Requirements for RTTs are frequently defined in relation to equipment numbers (23). On the other hand, recommendations for radiation oncologists more often refer to patient loads. This might also explain the workload developments observed in our study: As more patients per department are treated on a constant number of treatment units, the number of patients per RTT in growing. As RTTs and treatment units are both part of the radiation therapy process, other metrics such the number of fractions delivered per time period need to be included to account for evolving needs.

Most staffing recommendations also do not account for regional differences in the requirement for and availability of radiation therapy resources between countries. A large variability in radiation therapy resources between European countries is caused by epidemiological and economic disparities (20) and reflects the different roles and responsibilities of radiation therapy staff in different countries. Treatment planning and quality assurance might be carried out by medical physicists or, by varying degrees, by other radiation oncology professionals such as dosimetrists or RTTs, thereby decreasing the physicists' workload. Similarly, the involvement of radiation oncologists in the administration of chemotherapy varies considerably between European countries (23).

Several factors should be taken into account when evaluating the results of this study. The study population is more homogeneous than the overall profile of radiation oncology departments. A high proportion of the departments are academic centres (61\%), while only $31 \%$ of the participating departments were non-university public centres and $9 \%$ were private 
centres. Furthermore, many departments are located in high-income countries, which might explain the widespread availability of advanced treatment techniques. The availability of IMRT is correlated with gross national income, which translates into disparities in radiation therapy infrastructure among European countries (27). Countries might be over- or underrepresented depending on the proportion of the country's departments that are active EORTC ROG members.

While the EORTC's recommendations are periodically adjusted as a consequence of trends observed in the member departments, these trends themselves might be driven by updates to the staffing and workload recommendations. From our experience, recommendations by the EORTC or other organisations are used as benchmarks to optimize the organisation of radiation oncology departments and make investments according to current and predicted future needs. Notably, different national reimbursement strategies tend to have a major impact on the availability of radiation therapy resources and developments in departments (28).

An inherent limitation of our study is caused by the lack of specific data on techniques and actual task-related workload per profession, which are not collected by the FQ. Our workload estimations are based on patient numbers, which include both new and retreated patients. No data were available on the number of treatment fractions per patient and the applied radiation therapy techniques. Therefore, our assumption on changing workload per individual patient rely on the observed increasing availability of complex techniques and recent recommendations for hypofractionation in different types of cancer. Changing techniques may, however, impact the workload of different professions substantially, e.g. by the implementation of automation or sophisticated new tasks that are added to the workflow. Accurately measuring changing workload in the face of ever evolving technology is therefore increasingly challenging, though necessary.

Even though the FQ expires after two years or in case of significant changes of the simulation, treatment planning or delivery process, the EORTC does not strictly require the departments to update it unless a department applies to enrol patients into a new clinical trial. Therefore, some active member departments might have not been included in the current analysis, as they lack a valid FQ. The validity of the observed developments might further be limited because only 75 (43.4\%) of the departments were included twice, so both in the 2013 and 2019 analyses. In the pairwise analysis of the 75 departments that were available in 2013 and 2019, the patientworkload per radiation oncologist and medical physicist decreased significantly, while other changes were non-statistically significant.

However, the scale and regional diversity of our group in combination with the long period of continuous data collection emphasize the informative value of our study regarding 
developments of radiation oncology departments, particularly those in the academic clinical trials community throughout Europe and other regions.

In conclusion, growing patient numbers and the increasing complexity of radiation therapy techniques need to be counterbalanced by continuous adjustments of staffing and infrastructure in radiation oncology departments. More accurate, yet time-consuming, radiation therapy techniques become more widely available. The decreasing number of patients per medical physicist probably reflect the increasing utilization of more complex radiation therapy techniques, which rely on time-demanding treatment planning and frequent extensive quality assurance. The reduction of the number of treatment fractions per patient by hypofractionation and the use of SBRT and SRS as well as automation of parts of the radiotherapy workflow may explain the increasing number of patients per treatment unit and per RTT. Staffing and workload recommendations should be constantly adjusted to account for the proportion of complex treatments, courses and fractions delivered, to evolving fractionation schedules with changing time demands, varying professional roles and the responsibilities of all professions in radiation oncology departments to guarantee the safe implementation of new techniques to more accurately reflect the actual workload and local disparities.

\section{Conflicts of interest statement}

None declared.

\section{References}

1. Horiot JC, van der Schueren E, Johansson KA, Bernier J, Bartelink H. The programme of quality assurance of the EORTC radiotherapy group. A historical overview. Radiother Oncol. 1993;29(2):81-4.

2. Weber DC, Poortmans PMP, Hurkmans CW, Aird E, Gulyban A, Fairchild A. Quality assurance for prospective EORTC radiation oncology trials: The challenges of advanced technology in a multicenter international setting. Vol. 100, Radiotherapy and Oncology. 2011. p. 150-6.

3. EORTC Facility Questionnaire PDF version [Internet]. [cited 2020 Mar 11]. Available from: http://www.eortc.be/facilityquest/Facility Questionnaire.pdf

4. Minimum Requirements For Department Entering Patients In EORTC Radiation Oncology Group Clinical Trials [Internet]. [cited 2020 Mar 11]. Available from: http://www.eortc.be/facilityquest/

5. Bernier J, Horiot J, Bartelink H, Johansson K, Cionini L, Gonzalez Gonzal D, et al. Profile of radiotherapy departments contributing to the cooperative group of 
radiotherapy of the European Organization for Research and Treatment of Cancer. Vol. 34, Int. J. Radiat. 1996.

6. Budiharto T, Musat E, Poortmans P, Hurkmans C, Monti A, Bar-Deroma R, et al. Profile of European radiotherapy departments contributing to the EORTC Radiation Oncology Group (ROG) in the 21st century. Radiother Oncol. 2008 Sep;88(3):403-10.

7. Grant W, Hurkmans CW, Poortmans PM, Maingon P, Monti AF, Van Os MJH, et al. Quality assurance standards drive improvements in the profile of radiation therapy departments participating in trials of the EORTC Radiation Oncology Group. Radiother Oncol. 2014 Sep 1;112(3):376-80.

8. Fairchild A, Aird E, Fenton PA, Gregoire V, Gulyban A, Lacombe D, et al. EORTC Radiation Oncology Group quality assurance platform: Establishment of a digital central review facility. Radiother Oncol. 2012;103(3):279-86.

9. International Atomic Energy Agency (IAEA). DIRAC (DIrectory of RAdiotherapy Centres) [Internet]. [cited 2020 Sep 14]. Available from: https://dirac.iaea.org/

10. Otto K. Volumetric modulated arc therapy: IMRT in a single gantry arc. Med Phys. 2008;35(1):310-7.

11. Lievens Y, Grau C. Health Economics in Radiation Oncology: Introducing the ESTRO HERO project. Radiother Oncol [Internet]. 2012;103(1):109-12. Available from: http://dx.doi.org/10.1016/j.radonc.2011.12.026

12. Borras JM, Lievens Y, Barton M, Corral J, Ferlay J, Bray F, et al. How many new cancer patients in Europe will require radiotherapy by 2025? An ESTRO-HERO analysis. Radiother Oncol. 2016 Apr 1;119(1):5-11.

13. Shikama N, Tsujino K, Nakamura K, Ishikura S. Survey of advanced radiation technologies used at designated cancer care hospitals in Japan. Jpn J Clin Oncol. 2014;44(1):72-7.

14. Smith BD, Bellon JR, Blitzblau R, Freedman G, Haffty B, Hahn C, et al. Radiation Therapy for the Whole Breast: An American Society for Radiation Oncology (ASTRO) Evidence-Based Guideline Conflict of Interest Disclosure Statement. Pract Radiat Oncol [Internet]. 2018; Available from: https://www.practicalradonc.org/cms/10.1016/j.prro.2018.01.012/attachment/775de13 7-63cb-4c5d-a7f9-

95556340d0f6/mmc1.pdf\%0Ahttp://www.practicalradonc.org/cms/attachment/211910 5247/2089218135/mmc1.pdf

15. Morgan SC, Hoffman K, Andrew Loblaw D, Buyyounouski MK, Patton C, Barocas D, et al. Hypofractionated Radiation Therapy for Localized Prostate Cancer: An ASTRO, 
ASCO, and AUA Evidence-Based Guideline. Pract Radiat Oncol. 2018;

16. Videtic GM, Donington J, Giuliani M, Heinzerling J, Karas TZ, Kelsey CR, et al. Stereotactic Body Radiation Therapy for Early Stage Non-Small Cell Lung Cancer: an ASTRO Evidence-Based Guideline Conflict of Interest Disclosure Statement 2 ASTRO SBRT for Early Stage NSCLC Guideline. Pract Radiat Oncol [Internet]. 2017; Available from:

http://www.practicalradonc.org/cms/attachment/2097635109/2078316787/mmc1.pdf

17. Palma DA, Olson R, Harrow S, Gaede S, Louie A V, Haasbeek C, et al. Stereotactic ablative radiotherapy versus standard of care palliative treatment in patients with oligometastatic cancers (SABR-COMET): a randomised, phase 2, open-label trial. Lancet [Internet]. 2019 Apr;393(10185):2051-8. Available from: https://linkinghub.elsevier.com/retrieve/pii/S0140673618324875

18. Vaassen F, Hazelaar C, Vaniqui A, Gooding M, van der Heyden B, Canters R, et al. Evaluation of measures for assessing time-saving of automatic organ-at-risk segmentation in radiotherapy. Phys Imaging Radiat Oncol [Internet]. 2020;13(December 2019):1-6. Available from: https://doi.org/10.1016/j.phro.2019.12.001

19. McCarroll RE, Beadle BM, Balter PA, Burger H, Cardenas CE, Dalvie S, et al. Retrospective validation and clinical implementation of automated contouring of organs at risk in the head and neck: A step toward automated radiation treatment planning for low- And middle-income countries. J Glob Oncol. 2018;2018(4).

20. Lievens Y, Defourny N, Coffey M, Borras JM, Dunscombe P, Slotman B, et al. Radiotherapy staffing in the European countries: Final results from the ESTRO-HERO survey. Radiother Oncol. 2014 Aug 1;112(2):178-86.

21. Bentzen SM, Heeren G, Cottier B, Slotman B, Glimelius B, Lievens Y, et al. Towards evidence-based guidelines for radiotherapy infrastructure and staffing needs in Europe: The ESTRO QUARTS project. Radiother Oncol. 2005;75(3):355-65.

22. Slotman BJ, Cottier B, Bentzen SM, Heeren G, Lievens Y, Van Den Bogaert W. Overview of national guidelines for infrastructure and staffing of radiotherapy. ESTRO-QUARTS: Work package 1. Radiother Oncol. 2005;75(3):349.E1-349.E6.

23. Dunscombe P, Grau C, Defourny N, Malicki J, Borras JM, Coffey M, et al. Guidelines for equipment and staffing of radiotherapy facilities in the European countries: Final results of the ESTRO-HERO survey. Radiother Oncol. 2014 Aug 1;112(2):165-77.

24. INTERNATIONAL ATOMIC ENERGY AGENCY (IAEA). Staffing in Radiotherapy: An Activity Based Approach. Human Health Reports (CD-ROM) No. 
13. Vienna; 2015.

25. Evans S, Christofides S, Brambilla M. The European Federation of Organisations for Medical Physics. Policy Statement No. 7.1: The roles, responsibilities and status of the medical physicist including the criteria for the staffing levels in a Medical Physics Department approved by EFOMP Counci. Phys Medica [Internet]. 2016;32(4):533-40. Available from: http://dx.doi.org/10.1016/j.ejmp.2016.03.001

26. IPEM Guidelines for the Provision of a Physics Service to Radiotherapy [Internet]. [cited 2020 Mar 11]. Available from:

https://international.anl.gov/training/materials/BF/Publications/Physics staffing in RT Britain.pdf

27. Grau C, Defourny N, Malicki J, Dunscombe P, Borras JM, Coffey M, et al. Radiotherapy equipment and departments in the European countries: Final results from the ESTRO-HERO survey. Radiother Oncol. 2014 Aug 1;112(2):155-64.

28. Lievens Y, Defourny N, Corral J, Gasparotto C, Grau C, Borras JM, et al. How public health services pay for radiotherapy in Europe: an ESTRO-HERO analysis of reimbursement. Lancet Oncol. 2020;21(1):e42-54. 